\title{
Development of the constant force tool system with abrasive belt for grinding and polishing
}

\author{
Xin Wang ${ }^{1}$, Yan $\mathrm{Mu}^{2}{ }^{*}$, Huan $\mathrm{Liu}^{3}$, Xing-tian $\mathrm{Qu}^{4}$ and Xu Yang ${ }^{5}$ \\ School of Mechanical Science and Engineering, Jilin University, \\ Changchun, 130022, People's Republic of China

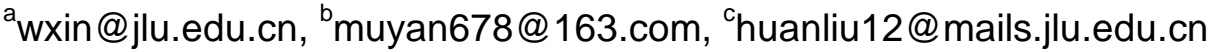

Keywords: Constant force polishing; Flexible polishing; Complex surfaces; Vibration; Simulation analysis.

Abstract. A constant force tool system for the abrasive belt grinding and polishing was developed in the paper. With the addition of the constant force control during the finishing of curved surfaces, the accuracy and the surface roughness could be improved. The constant force tool system would be used on the hybrid machine tool, and was composed by the following function modules, the abrasive belt system, the detection and compensation unit of the grinding force, and the vibration isolation system. With the statics simulation, harmonic response analysis and modal analysis of the tool system were carried out. All results showed that the design was reasonable and effective.

\section{Introduction}

The finishing machining of complex surfaces was a technology with a great quantity of advanced theories and complicated machining processes which was widely applied in machining process. Particularly, abrasive belt polishing had the characteristics of high efficiency, low grinding heat, elastic grinding and high polishing quality, so it was widely regarded as a kind of high efficiency and low energy consumption mechanical manufacturing technology with flexible craft and wide adaptability $[1,2]$. Owning to the advantage of low cost, high stiffness and quick response, the traditional passive grinding and polishing tools was widely used. But the problem of single machining object, rigid shock and vibration would appear with the process $[3,4]$, which affects the workpiece dimension error, surface roughness and the waviness [5,6]. The constant force tool system for grinding and polishing was used in the independent development blade hybrid polishing machine [7]. Based on the structure characteristic of the hybrid machine and the movement way of the tool system, the constant force tool system with abrasive belt for grinding and polishing was designed, which could realize the functions of flexible polishing, vibration isolation and variable tension.

\section{The principle and structure of the constant force tool system}

To realize the constant force polishing for variable curvature of surfaces, the designed polishing tool system mainly contained three subsystems: the polishing system composed of the drive motor, the tensioning mechanism and the abrasive belt wheel train, the polishing force detection and compensation system composed of the cylinder force transducer and the low friction pair, the motor vibration isolation system composed of the damper bracket and the spring damper.

As shown in Fig.1, the driving wheel of abrasive belt system was driven by the main motor, the contact wheel of the polishing head can rotated by the abrasive belt under the action of tension mechanism. In the tension mechanism, the idler which contacted with the abrasive belt was pushed by the cylinder module. The tension of the abrasive belt could be adjusted after the cylinder module motion at a set force. The guide column was a part of guide device, which guided the support plate to move along the column in the vertical direction. The normal polishing force was measured by the force transducer, and the measurement data was fed back to the computer to compare with the set value. The grinding and polishing with constant force could be realized after the adjustment of the electric proportional valve and the force compensation of low friction pair cylinder. In addition, in order to 
grind and polish the complicated surfaces, the change wheel motor drove four different curvature contact wheels of the polishing head, adapting to the curvature of the component surface. Then the quality and efficiency of the polishing process could be both guaranteed.

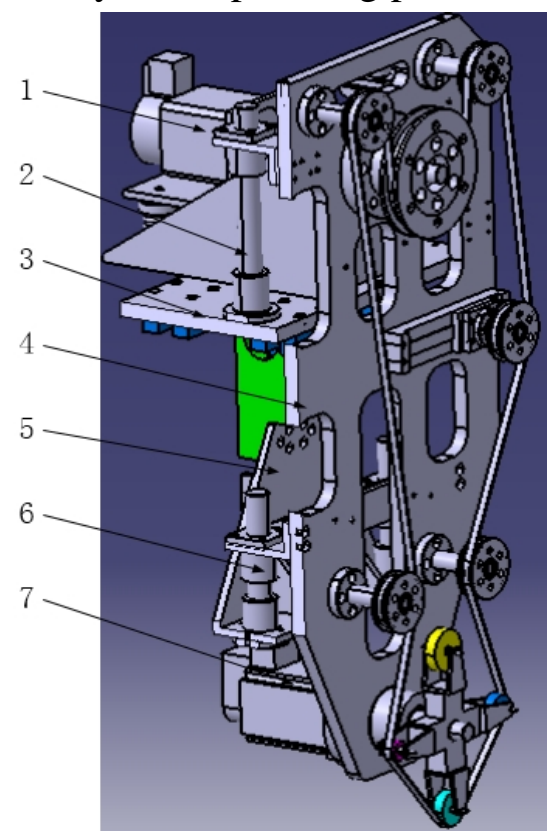

(a)

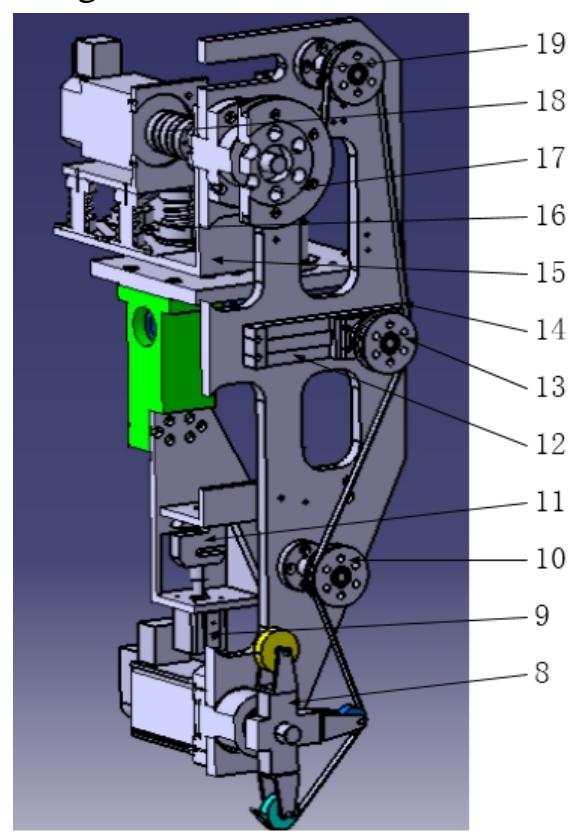

(b)

1-Polishing motor 2-Guide column 3-Movable cross-beam 4-Wheel train support plate 5-Support Plate 6-Linear bearing 7- The change wheel motor

8-The complex curvature of polishing head 9-Low friction cylinder 10-Idler 11-Force transducer 12-Cylinder module13-Tension mechanism 14-Abrasive belt 15-Damper bracket16-Spring-Damper 17-Driving wheel 18-Coupling 19-Idler shaft

Fig.1 Basic structure of the constant force tool system grinding and polishing

\section{The design of tool system}

According to the need of the hybrid machine tool for processing of blade, the maximum polishing speed $V_{s}$ in machining process was set to $20 \mathrm{~m} / \mathrm{s}$, the workpiece feed speed $V_{w}$ was $0.2 \mathrm{~m} / \mathrm{min}$, the maximum polishing depth $a_{p}$ was set to $0.1 \mathrm{~mm}$, the specific grinding energy $U_{S}$ was $1500 \mathrm{~kg} / \mathrm{mm}^{2}$, the width $B$ of the abrasive belt was set to $15 \mathrm{~mm}$, and the power factor was 0.7 . The tangential force of abrasive belt for grinding and polishing was $F_{t}$, the normal force was $F_{n}$ and the motor power was $P$, which could be calculated as follows [8].

$$
\begin{aligned}
& F_{t}=U_{S} \frac{V_{w}^{0.84}}{V_{s}} a_{p}^{0.71} B_{。} \\
& F_{n}=U_{s} \frac{V_{w}^{0.84}}{V_{S}} a_{p}^{0.68} B_{o} \\
& P=F_{t} V_{s}=\frac{U_{s} V_{w}^{0.84} V_{s}^{0.15} a_{p}^{0.68} B}{\eta}
\end{aligned}
$$

The tensioning mechanism was necessary to ensure the stable polishing of the abrasive belt tool system in the machining process. If the tension force was set suitably, the problems of slippage and running deviation could be avoided. The minimum tension $F_{\text {amin }}$ provided by cylinder module was calculated as Eq. 4[8]. 


$$
\mathrm{F}_{\alpha \min }=\mathrm{F}_{\mathrm{t}} \frac{\alpha \cos \gamma}{\mathrm{e}^{\mu \alpha}-1}=\frac{\mathrm{F}_{\mathrm{n}}}{\xi} \frac{\alpha \cos \gamma}{\mathrm{e}^{\mu \alpha}-1}
$$

where $\mu$ was the friction coefficient between the abrasive belt and the tension wheel and $\mu=0.35 . \gamma$ was the angle between the abrasive belt loose edge and the connecting line of the tension wheel and the contact wheel, and $\gamma$ was set to $22^{\circ} ; \alpha$ was the wrap angle of the abrasive belt on the drive wheels , which was set to $200^{\circ}, \xi$ was the ratio of the normal force and the tangential force and it was usually taken to 2[8].

In the grinding process of the abrasive belt, the vibration would affect the quality of workpiece surface. In order to avoid the impact of the main motor vibration in the machining process, the spring and damping structure was designed to realize the motor vibration isolation in this paper. As shown in Fig. 1, the polishing motor 1 was fixed on the damper bracket 15 through four spring dampers 16 . The vibrations caused by the polishing motor 1 would be reduced to the minimum with the help of spring damper. The transmissibility $\alpha$ was expressed by the ratio of the damper bracket. The relationship between amplitude of the force $X$ and the amplitude of polishing motor force $A$ could be expressed as Eq. 5 where the frequency $\omega$ of the polishing motor vibration was $25 \mathrm{~Hz}$. The vibration frequency $\omega_{n}$ of the support frame was $6.4 \mathrm{~Hz}$. The damping factor was 0.2 .

$$
\alpha=\frac{F_{D B}}{F_{P M}}=\sqrt{1+\left(2 \zeta \frac{\omega}{\omega_{n}}\right)^{2}} / \sqrt{\left[1-\left(\frac{\omega}{\omega_{n}}\right)^{2}\right]^{2}+\left[\left(2 \zeta \frac{\omega}{\omega_{n}}\right)^{2}\right]} \text { 。 }
$$

Based on the above formula, the relationship between the transmissibility and the vibration frequency of the motor could be showed in an intuitive way in Fig. 2.

The transmissibility value was $0.1296 \mathrm{MPa}$. With the application of the ANSYS harmonic response analysis, constraints on the motor vibration isolation system was added. The stress of $1 \mathrm{MPa}$ was applied on the polishing motor and the amplitude of stress of the damper bracket was $0.16 \mathrm{MPa}$ when the vibration frequency of the motor was $25 \mathrm{~Hz}$. This could be seen in Fig. 3. The calculation and simulation results showed that the vibration isolation was efficient.

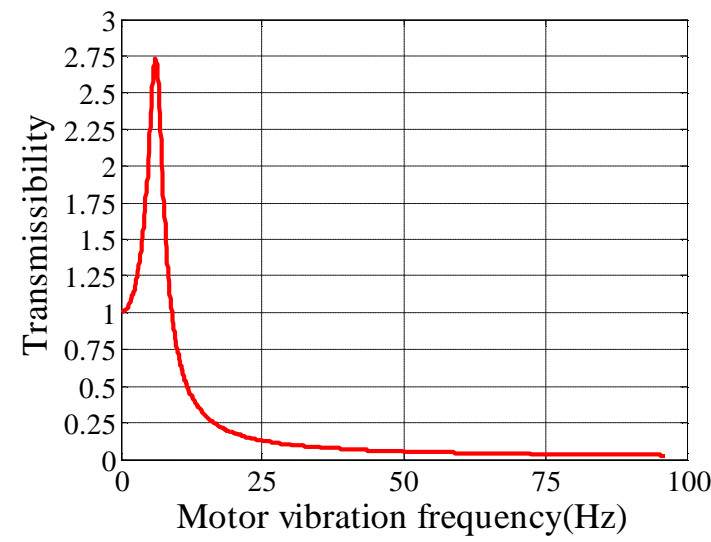

Fig.2.The transmissibility value

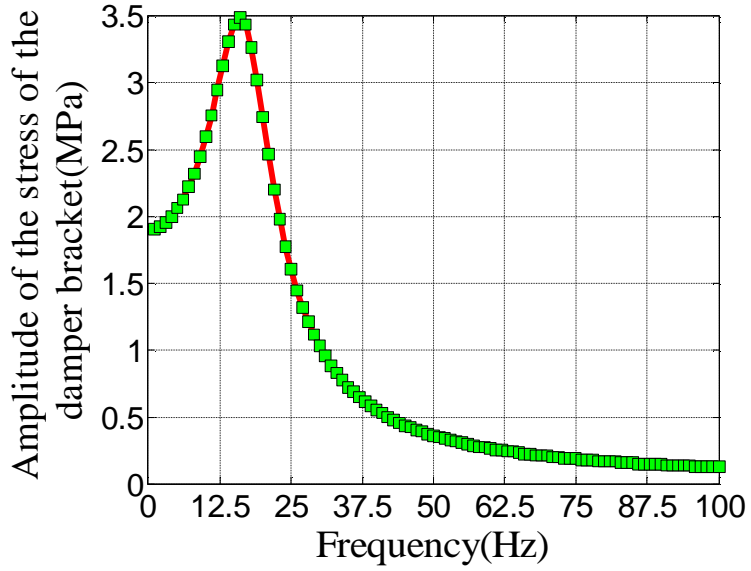

Fig. 3.The amplitude of the damper bracket

The polishing wheel was pushed by the constant force system with the set pressure, and contacted with the blade tightly. The air medium compressible characteristics, which was the certain amount floating allowance, ensured cylinder to avoid owing cuts, over cuts, negative rigidity shock and vibration phenomenon. The application of pneumatic constant force grinding and polishing tools system could realize the objective of low surface residual stress, low surface roughness and foliar complete blade. The detection and compensation mechanism of the grinding and polishing force was composed of the low friction cylinder and the force transducer. 


\section{The finite element simulation of the tool system}

In order to guaranteed the designed tool system to satisfy the requirement of stiffness and strength in the process, the linear statics structure analysis of the tool system was simulated by using the Workbench module of software of ANSYS.

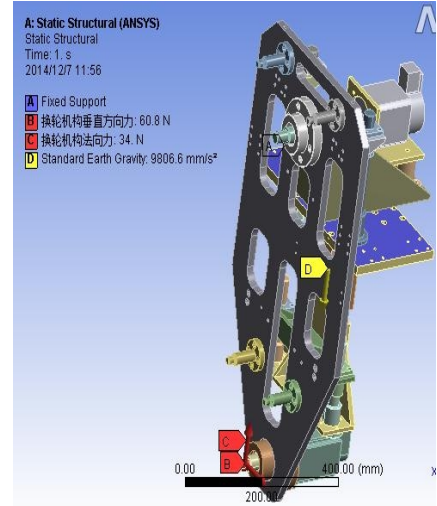

(a) Applied load

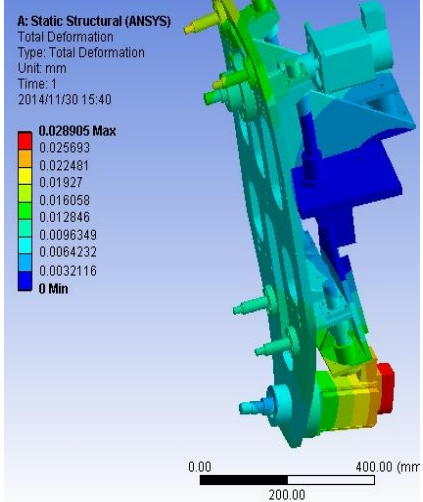

(b) System deformation

Fig.4. Statics structure analysis

Fig. 4 (a) showed the work conditions after setting the meshing model in ANSYS. After the repeated correction on the system structure, the final train and stress analysis could be obtained which are shown in Fig. 4(b).

Besides the static load, the external vibration load and the vibration load of the system had important influence on the constant force tool system. The modal analysis was carried out on the structure to avoid the resonance effect between the drive motor and the tool system.

After the simulation analysis of the system, the deformation trend and the six-mode shape had been obtained, which was shown in Fig. 5 and Table 1. It was obvious that the resonance phenomenon would not occur.

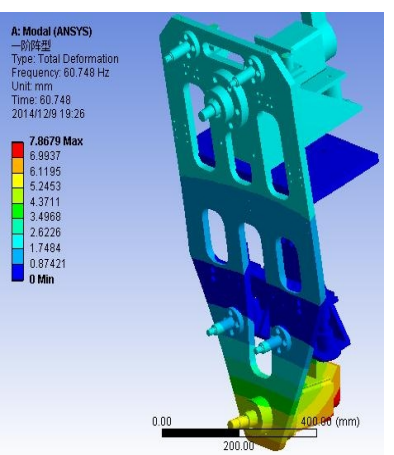

(a) The first model

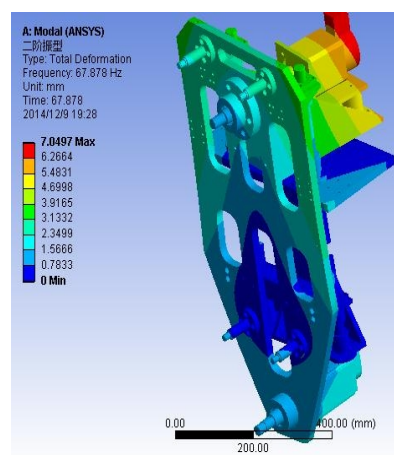

(b) The second model

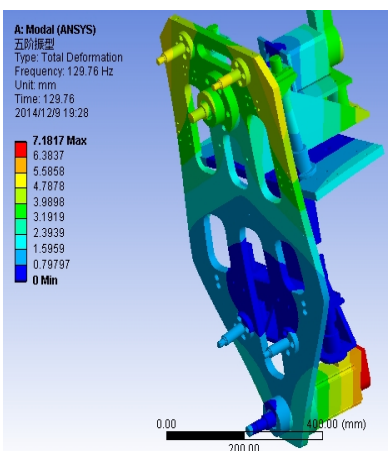

(e) The fifth model

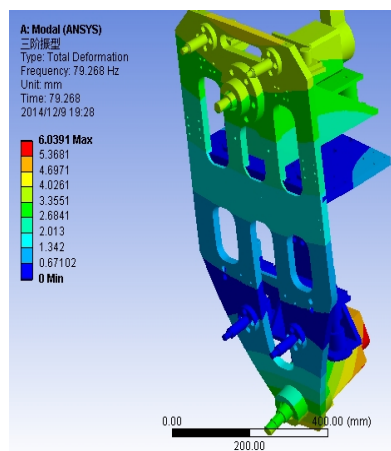

(c) The third model

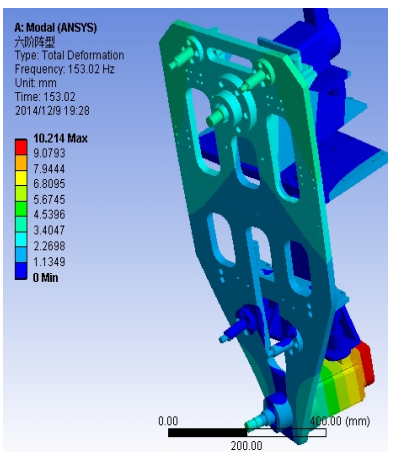

(f) The sixth model

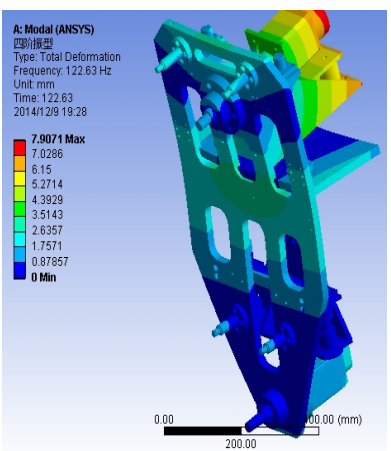

(d) The fourth model

Fig.5. Model shape 
Table 1. Structural modal analysis result

\begin{tabular}{c|cccccc}
\hline Order & 1 & 2 & 3 & 4 & 5 & 6 \\
\hline Frequency $[\mathrm{Hz}]$ & 60.748 & 67.878 & 79.268 & 122.63 & 129.76 & 153.02 \\
\hline
\end{tabular}

As shown from Table 1, the range of the inherent frequency of the tool system is from $60 \mathrm{~Hz}$ to $153 \mathrm{~Hz}$. Also, each of the system modal frequencies doesn't has a multiple relation with the motor vibration frequency, which is $25 \mathrm{~Hz}$. Consequently, the resonance phenomenon can ${ }^{t}$ be happened. The calculated results and the simulation analysis shown above support a fact that the system has a good dynamic characteristic.

And all of the simulation and experiment above were based on the machine tool as shown in Fig.6.

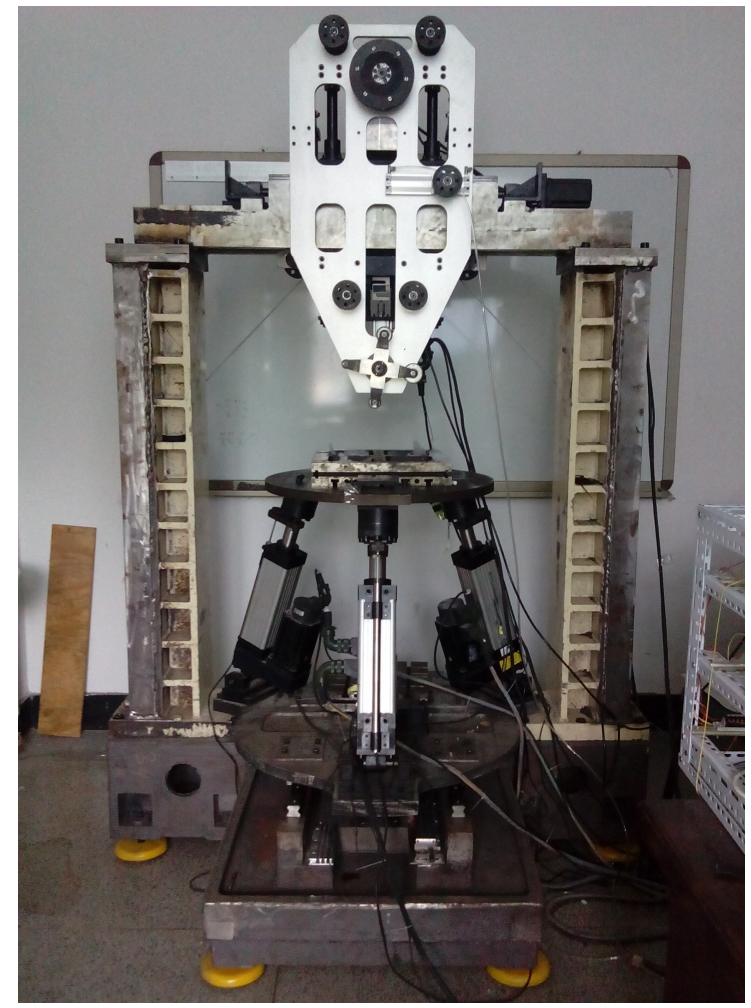

Fig.6.The constant force machine tool with abrasive belt for grinding and polishing

\section{Summary}

(1) During the design of the constant force tool system with abrasive belt for grinding and polishing, the factors of the tension force, the vibration and the flexible polishing force were considered. We systematically solved the problems of the belt breakage, the vibration of the machining surfaces and the rigid impact.

(2) Considering the machine body and the characteristics of the tool system, the tool system for polishing and grinding was reasonably designed to guarantee the assembly process and the rational distribution.

(3) With the simulation analysis of the polishing tool system, the aspects of system stiffness, strength and modal were checked and corrected to make the system obtain the good ability of deformation resist, damage resistance and anti- resonance. 


\section{Acknowledgments}

The authors would like to be grateful to a grant from the National Nature Science Foundation of China (Grant No.51135006).

\section{References}

[1] Sung-San Cho, Yong-Kyoon Ryu, Seung-Young Lee, Curved surface finishing with flexible abrasive tool, International Journal of Machine Tools and Manufacture, January (2002)229-236.

[2] Ioan D. Marinescu, W. Brian Rowe, Abrasives and abrasive tools, Tribology of Abrasive Machining Processes, (2013)243-311.

[3] B. Rasmussen, S. Derby, Design and evaluation of robotic end-effectors for an automated die finishing system, in: Proceedings Symposium on Computer Aided Design and Manufacture of Dies and Molds. ASME Winter Annual Meeting, (1988)61-74.

[4] Liao liang, Xi Feng-feng (Jeff), Liu Ke-fu, Modeling and control of automated polishing debur ring process using a dual-purpose compliant toolhead, International Journal of Machine Tools and Manufacture, (2008) 1454-1463.

[5] Jiang Zhou-da, Advances of research on the character of grinding chatter, Manufacturing Technology \& Machine Tool, (2012)35-42.

[6] Duan Ji-hao, Shi Yao-yao, Zhang Jun-feng, Dong ting, Li Xiao-biao, Flexible polishing technology for blade of aviation engine, Acta Aeronautica Sinica, (2012)573-578.

[7] Lin Jie-qiong, Zhao Ji, Wang Yi-qiang, Zhu Pei-xing, Polishing die free form surface by series and parallel polishing machine, Journal of Jilin university (Engineering and Technology Edition), (2006)209-212.

[8] Huang Yun, Huang Zhi. The Technology and the Enginneering Application of Modren Abrasive Belt Grinding. Chongqing University Press. 2009. 